\title{
Self Esteem and Disordered Eating Attitudes in Female Adolescents
}

\author{
Meghna Basu Thakur \\ Jyotika Varmani ${ }^{2}$ \\ ${ }^{1}$ Professor and Head, Department of Psychology, R.D. National College, Bandra, Mumbai. \\ ${ }^{2}$ Visiting Faculty, Department of Psychology, R.D. National College, Bandra, Mumbai. \\ E-mail - meghnabasuthakur@yahoo.com
}

\section{ABSTRACT}

Eating disorders are largely prevalent in a modern era. These disorders originate overtime from the attitudes that individuals hold about eating. Adolescent girls are found to be especially vulnerable when it comes to beliefs about body shape and appearance. The present study looks at self-esteem as a factor in determining disordered eating attitudes. Data obtained from sixty adolescent girls $(N=60)$ ranging from the ages 16-17 is analysed. Results show the significant relationship between selfesteem and disordered eating attitudes $(t=3.23, p<.001)$. The results are discussed with respect to the influence of media and religious practices in the eating beliefs of adolescent girls, and the growing influence of the Western culture. The implications of the study in terms of intervention for uplifting self-esteem and suggestions for future research are also presented.

Key words: self esteem, eating disorders, disordered eating attitudes, adolescence, eating attitudes.

\section{INTRODUCTION}

Eating attitudes are defined as beliefs, thoughts, feelings, behaviours and relationship with food [1]. Eating attitudes are the foundation of the development of eating disorders. Healthy eating attitudes contribute to healthy living [2]. There are several factors that determine eating disorders. Over years of research, self-esteem has emerged as an important factor. Early research has established that problems in self-image, in other words, self-esteem, precede the onset of eating disorders [3]. Low self-esteem in the formative years has been found to predict disordered eating behaviours in later life in classic studies [4] and in more recent research [5]. Some early studies have established self-esteem as a mediator between body satisfaction and disordered eating attitudes [6-7]. Yet, there have been contrary results regarding this relationship [8-9].

\section{Disordered Eating Attitudes, Self-esteem and Age}

Adolescence is characterized with marked changes in school life, friendships, family relations, one's self-concept and goals for one's future. These changes have a definite impact on the self-esteem of an adolescent, and consequently 
on other factors. Low self-esteem in particular has been associated to unhealthy eating behaviours [10-11]. In research on self-esteem and overeating, low self-esteem has been linked to overeating and consequently, excessive weight in adolescents, even if body mass index (BMI) is controlled for [12]. A definite body type is generally glorified in the media i.e. slenderness [13]. Adolescents are especially vulnerable to the impact of the media and social media [14]. This kind of media portrayal coupled with low selfesteem is a definite risk factor for disordered eating attitudes in this population.

\section{Disordered Eating Attitudes, Self-esteem and Gender}

A review of literature shows that self-esteem is generally found at significantly lower levels in girls than in boys [15]. It also decreases progressively from early to late adolescence [16]. In a noteworthy prospective study, researchers [3] studied self-esteem and vulnerability to eating disorders in adolescent females. He found that low self-esteem correlated to a concern for growing fat and other psychological problems. More significantly, he found that girls with lower self-esteem at a younger age were at a heightened risk of developing eating disorders at an older age. Eating disorders themselves have a strong association to gender. They are more prevalent in females especially in adolescent girls owing to this population's preoccupation with body shape, weight and diet [17]. Females are found to be more prone to engage in maladaptive eating behaviours like binge eating, dieting, and vomiting [18]. The indulgence of this population in dieting and other weight loss strategies is associated to a growing risk of eating disorders [17].

\section{Disordered Eating Attitudes, Self-esteem and Culture}

Several ethnic and racial studies have been conducted in this area. These studies have found a definite relation between low self-esteem and maladaptive eating behaviours [19]. It is also understood that low self-esteem is not necessarily linked to disordered eating attitudes universally. Such associations will definitely be stronger in ethnic and racial groups that emphasize body shape and appearance. It is common knowledge that western cultures place a strong emphasis on physical appearance. Females in these cultures are found to associate attractiveness to being thin [20]. Several Indian studies have established that adolescent girls in the country show similar ideals as that of girls in Western cultures with respect to body image disturbance and eating disorders [21-22]. The increasing exposure to Western media and fast paced globalization has further increased the presence of eating disturbance, leading to high levels of body dissatisfaction and a fear of being overweight [22]. There is however, a lack of research tying self-esteem to disordered eating attitudes in female adolescents in the Indian context.

Previous research has not successfully established a strong link between low self-esteem and disordered eating attitudes. Also, there has been a major focus on either the relation between obesity and self-esteem or between eating behaviours and self-esteem. Thus, the present study attempts to singularly establish a relation between self-esteem and the precursor of eating disorders, that is, disordered eating attitudes. Also, this relationship is explored in the present study with a sample of Indian adolescents, thus checking for the strength of this relationship with regards to the Indian context. 


\section{METHODOLOGY}

The main hypothesis of the study was that participants with disordered eating attitudes will have lower self-esteem scores on the Rosenberg Self-Esteem Scale than participants not showing disordered eating attitudes. The sample comprised of sixty female adolescent girls studying in different junior colleges were selected based on availability $(\mathrm{N}=60)$. Their ages ranged from 16 to 17 years.

\section{Scales Used}

Disordered Eating Attitudes: The Disordered Eating Attitude Scale (English Version) was used for the purpose of the study. The internal consistency of the scale has been established as 0.76 . The scale has been found to have appropriate internal consistency, convergent validity and test-retest reliability and is useful in measuring eating attitudes in English speaking countries [1].

Self Esteem : The Rosenberg self-esteem scale was developed Morris Rosenberg in 1965. It is a ten-item Likert-type scale. The answers for every item range on a fourpoint scale - from strongly agree to strongly disagree. The scale has half its items positively worded and half negatively worded. The scale measures self-esteem as a state rather than a trait and hence was selected for the purpose of the present study that seeks to measure self-esteem disordered eating attitudes in a particular stage in life, namely, adolescence. The Rosenberg self-esteem scale is an established measure of selfesteem with a reliability ranging from 0.5 to 0.9 depending on the context in which the instrument is used. The validity of the scale is found to range from 0.77 to 0.88 [23].

Written informed consent regarding the study was obtained from the 60 participants. The scales were then administered and the participants were orally debriefed about the purpose of the study. The obtained data was subject to analysis.

\section{Statistical Analysis}

Data was analysed using SPSS 20. Any participant scoring more than 90 on the DEAS was considered to have a disordered eating attitude. Thus, two groups were identified. One showing disordered eating attitudes $(\mathrm{N}=21)$ and the other not showing disordered eating attitudes i.e. the control group $(\mathrm{N}=39)$ i.e. group 1 and group 2 respectively. Looking at the different sample sizes, a $t$ test assuming unequal variances was conducted to compare the means of self-esteem scores of the groups.

\section{RESULTS}

Table 1: Means, SDs and t-value of the two groups

\begin{tabular}{|c|c|c|c|}
\hline Group & Mean & SD & t-value \\
\cline { 1 - 3 } & 1.70 & 0.23 & $3.23^{* *}$ \\
\cline { 1 - 3 } & 2.30 & 0.47 & $\mathrm{P}<0.01$ \\
\hline
\end{tabular}

**significant

Both groups were well matched socio-demographically in all respects. Table 1 shows that group $1(M=1.70 ; S D=0.23)$ showed lower scores on the Rosenberg Self Esteem Scale than group $2(M=2.30 ; S D=0.47)$. This difference was found to be significant $(t=3.23$; $p<0.01)$. 


\section{DISCUSSION}

The above results clearly indicate that self-esteem is one of the antecedents of disordered eating attitudes for adolescent girls even in the Indian context. The high prevalence of disordered ideas about eating indicates that the common media portrayal of ideal eating practices and emphasis on being slim at the cost of healthy nourishment needs to be modified. The idealization of Western practices also play a role Adolescents need education on healthy eating rather than on losing weight. Since self-esteem is identified as precedent, intervention programs can target adolescents with low self-esteem and help them build on this deficit to prevent the development of disordered eating attitudes in this group. The present study has shown the link between low self-esteem and disordered eating attitudes. Further research can look into other factors along with self-esteem that have a significant impact on eating attitudes.

\section{REFERENCES}

1. Sallet PC, de Alvarenga PG, Ferrão $Y$, de Mathis MA, Torres AR, Marques A, Fleitlich-Bilyk B. Eating disorders in patients with obsessive-compulsive disorder: prevalence and clinical correlates. Int J Eating Disord 2010;43(4):315-25.

2. Naughton $P$, McCarthy S, McCarthy M. Healthy eating attitudes and healthy living: An examination of the relationship between attitudes, food choices and lifestyle behaviours in a representative sample of Irish adults. Proc Nutr Soc 2013;(72):113-8.

3. Button EJ. Self-esteem in girls aged 11-12: Baseline findings from a planned prospective study of vulnerability to eating disorders. J Adolesc 1993;13:407-13.

4. Fryer S, Waller G, Kroese BS. Stress, coping and disturbed eating attitudes in teenage girls. Int J Eating Disord 1997;22:427-36.

5. Polivy J, Herman CP. Sociocultural idealization of thin female body shapes: An introduction to the special issue on body image and eating disorders. J Soc Clin Psychol 2004;23(1):16.

6. Vitousek KB, Hollon SD. The investigation of schematic content and processing in the eating disorders. Cogn Ther Res 1990;14:191-214.

7. Fairburn, CG, Shafran R, Cooper Z. A cognitive behavioral theory of anorexia nervosa. Behav Res Therapy 1999;37:1-13.

8. van den Berg P, Thompson JK, Obremski-Brandon K, Coovert M. The Tripartite Influence Model of body image and eating disturbance: A covariance structure modeling investigation testing the mediational role of appearance comparison. Journal of Psychosom Res 2002;53:1007-20.

9. Heywood S, McCabe MP. Negative affect as a mediator between body dissatisfaction and extreme weight loss and muscle gain behaviors. J Health Psychol 2006;11(6):833-44.

10. Cartwright M, Wardle J, Steggles N, Simon AE, Croker H, Jarvis MJ. Stress and Dietary Practices in Adolescents. Health Psychol 2003;22:362-9.

11. Sjöberg RL, Nilsson KW, Leppert J. Obesity, shame, and depression in school-aged children: a population-based study. Pediatrics 2005; 116:389-92.

12. Ackard DM, Croll JK, Kearney-Cooke A. Dieting frequency among college females: association with disordered eating, body image, and related psychological problems. J Psychosom Res 2002;52:129-36.

13. Smolak L. Body image development in childhood. In TF Cash \& L Smolak (Eds.), Body image: A handbook of science, practice, and prevention (2nd ed., pp. 67-75). New York: Guilford Press ; 2011.

14. Brown GW, Bifluco A, Andrews B. Self-esteem and Depression: Aetiological issues. Soc Psych Psychiatr Epidemiol 1990;25:235-43. 
15. Robins RW, Trzesniewski KH. Self-esteem development across the lifespan. Curr Dir Psychol Sci 2005;14:158-62.

16. O'Dea JA Self-concept, self-esteem and body weight in adolescent females: a three-year longitudinal study. J Health Psychol 2006;11:599-611.

17. Burgic M, Burgic S, Gavric Z. Eating Attitudes In Adolescent Girls. Psychiatr Danub 2010;22(2):298-300.

18. McCabe MP, Riccciardelli LA. Parent, peer, and media influences on body image and strategies to both increase and decrease body size among adolescent boys and girls. Adolescence $2001 ; 36: 225-40$.

19. Strauss RS. Childhood obesity and self-esteem. Pediatrics 2000;105:e15.

20. Furnham A, Badmin N, Sneade I. Body image dissatisfaction: Gender differences in eating attitudes, self-esteem, and reasons for exercise. J Psychol 2002;136:581-96.

21. King MB, Bhugra D. Eating disorders: lessons from a cross-cultural study. Psychol Med $1989 ; 19(4): 955-8$.

22. Gupta MA, Chaturvedi SK, Chandarana PC, Johnson AM. Weight-related body image concerns among 18-24-year-old women in Canada and India: An empirical comparative study. J Psychosom Res 2001;50(4):193-8.

23. Robins RW, Hendin HM, Trzesniewski KH. Measuring global self-esteem: Construct validation of a single-item measure and the Rosenberg Self-Esteem Scale. PersonalSoc Psychol Bull 2001;27(2):151-61.

Acknowledgements - Nil

Conflict of Interest - Nil

Funding - Nil.

\section{CALL FOR PAPERS - INDIAN JOURNAL OF MENTAL HEALTH (2015)}

The Indian Journal of Mental Health publishes papers in various areas of psychology, psychiatry, mental health and psychiatric social work. We hereby request interested authors to send in their papers earliest by September $1^{\text {st }}$ for the next issue (July-December 2015) of the journal. The submitted papers must be in the required format as per the journal guidelines for authors (see at the end of this issue or visit the website). Submissions not in the journal format shall be returned for formatting. Review articles, original research papers, case reports, single case psychotherapy experiences, viewpoints on critical issues in mental health, poems related to mental health, innovative therapies in mental health and ethical dilemma papers are invited. Please submit all papers via email to the editor at avinashdes888@gmail.com

Please note that all papers shall be screened for plagiarism using computerized software and if found guilty of the same, shall be duly informed with due action taken as per advice of the journal's editorial board. 